\title{
MAGNETIC FIELDS IN RADIO-QUIET QUASARS
}

\author{
R. SCHLICKEISER, A. CRUSIUS-WATZEL \\ Max-Planck-Institut für Radioastronomie \\ Auf dem Hügel 69 \\ 5300 Bonn 1, F.R.G.
}

As an hypothesis the sharp far-infrared turnovers in the spectra of several radio-quiet galactic nuclei [1-3] are attributed to the modifications of synchrotron emission arising from the presence of a thermal background plasma. We calculate the synchrotron emission from a powerlaw distribution of relativistic electrons $N(y)=N_{0} \gamma^{-s}$ in a large-scale random magnetic field of strength $B$ embedded in a thermal plasma of density $n_{e}$. Two major modifications of the classical vacuum theory of synchrotron emission are established [4]:

A) synchrotron sources can be optically thick only in a small frequency range around the Razin-Tsytovich frequency, $\nu_{R}=20\left(\mathrm{n}_{\mathrm{e}} / \mathrm{cm}^{-3}\right)(\mathrm{B} / \mathrm{G})^{-1}$ $\mathrm{Hz}$, whereas at smaller and higher frequencies the sources are optically thin;

B) at frequencies above $\nu_{R}$ the synchrotron intensity in a plasma behaves exactly the same way as in the vacuum case, $I\left(v>\nu_{R}\right) \propto v^{-\alpha}, a=$ $(s-1) / 2$, whereas at frequencies below $\nu_{R}$ the intensity is exponentially reduced, $I\left(\nu<\nu_{R}\right) \propto \exp \left(-\nu_{R} / \nu\right)$.

Applying these findings to the observations we find:

1) the low-frequency exponential cutoff below the Razin-Tsytovich frequency provides an excellent fit to the observed spectra from radio-quiet quasars, if $v_{R} \simeq 2 \cdot 10^{12} \mathrm{~Hz}$. The value of $v_{R}$ suggests the relation $B=$ $10^{-11}\left(\mathrm{n}_{\mathrm{e}} / \mathrm{cm}^{-3}\right) \mathrm{G}$ between the magnetic field strength and the plasma density in these objects;

2) none of the existing observations [1-3] are incompatible with the synchrotron origin of the emission.

\section{References}

[1] Engargiola, G., Harper, D.A., Elvis, M. and Willner, S.P. (1988) Astrophys. J. 332, L19-L22.

[2] Edelson, R.A., Gear, W.K.P., Malkan, M.A. and Robson, E.I. (1988) Nature 336, 749-751.

[3] Chini, R., Kreysa, E. and Biermann, P.L. (1989) Astron. Astrophys. 219, 87-97.

[4] Schlickeiser, R. and Crusius, A. (1989) IEEE Trans. Plasma Sci. 17, 245-251. 